\title{
Work as an external quantum observable and an operational quantum work fluctuation theorem
}

\author{
Konstantin Beyer, ${ }^{*}$ Kimmo Luoma, ${ }^{\dagger}$ and Walter T. Strunz (i) $^{\dagger}$ \\ Institut für Theoretische Physik, Technische Universität Dresden, D-01062, Dresden, Germany
}

\begin{abstract}
(Received 21 March 2020; revised 14 August 2020; accepted 10 September 2020; published 28 September 2020)
\end{abstract}
\begin{abstract}
We propose a definition of externally measurable quantum work in driven systems. Work is given as a quantum observable on a control device which is forcing the system and can be determined without knowledge of the system Hamiltonian $\hat{H}_{\mathcal{S}}$. We argue that quantum work fluctuation theorems which rely on the knowledge of $\hat{H}_{\mathcal{S}}$ are of little practical relevance, contrary to their classical counterparts. Using our framework, we derive a fluctuation theorem which is operationally accessible and could in principle be implemented in experiments to determine bounds on free energy differences of unknown systems.
\end{abstract}

DOI: 10.1103/PhysRevResearch.2.033508

\section{INTRODUCTION}

The classical Jarzynski equality (JE) [1,2] connects the work $W$ performed in nonequilibrium realizations of a driving protocol with the equilibrium free energy difference $\Delta F$ by the relation

$$
\left\langle e^{-\beta W}\right\rangle=e^{-\beta \Delta F}
$$

The driving protocol is given by a time-dependent system Hamiltonian $H_{\mathcal{S}}(t)$. Initially, the system is in a thermal state with respect to the initial Hamiltonian $H_{\mathcal{S}}^{A}=H_{\mathcal{S}}(0)$ at inverse temperature $\beta$. At the end of the protocol the final Hamiltonian $H_{\mathcal{S}}^{B}=H_{\mathcal{S}}(T)$ is reached. The JE tells us that, by measuring the work $W$ for many realizations of the protocol, we can obtain $\Delta F=-1 / \beta \ln \left(Z_{B} / Z_{A}\right)$ between the thermal states of $H_{\mathcal{S}}^{A}$ and $H_{\mathcal{S}}^{B}$ even though the system never reaches the thermal state with respect to the final Hamiltonian.

The JE has been celebrated not only for its theoretical impact on the understanding of nonequilibrium dynamics but also because of its practical relevance for the estimation of free energy differences of complex mesoscopic systems [3-16]. The prototypical example is the forced unfolding of molecules such as RNA $[6,7,9,11,12]$. The work needed to expand the molecule is determined externally by pulling one end and simultaneously measuring the force during this process (e.g., with an AFM). It is then possible to determine $\Delta F$ without knowledge about the details of the system of interest (e.g., the Hamiltonian of the molecule) and without waiting until the system has reached a thermal state.

\footnotetext{
*konstantin.beyer@tu-dresden.de

†kimmo.luoma@tu-dresden.de

‡walter.strunz@tu-dresden.de
}

Published by the American Physical Society under the terms of the Creative Commons Attribution 4.0 International license. Further distribution of this work must maintain attribution to the author(s) and the published article's title, journal citation, and DOI.
Extending the concept of work to the quantum case has led to different proposals [17-26]. A particularly popular approach is the so-called two-point measurement (TPM) scheme. One of the reasons for its broad acceptance [4,27-32] is its ability to reproduce all kinds of fluctuation relations known from classical statistical physics, especially the JE (1). The standard TPM scheme is based on two projective energy measurements at time $t=0$ and $t=T$. Assuming a finite quantum system, the observables are given by $\hat{H}_{\mathcal{S}}^{A}=$ $\sum_{a} E_{a}|a\rangle\langle a|$ and $\hat{H}_{\mathcal{S}}^{B}=\sum_{b} E_{b}|b\rangle\langle b|$, where the $|a\rangle$ 's and $|b\rangle$ 's are the energy eigenstates.

Throughout this article we will consider a unitary system evolution $\mathrm{U}=\mathcal{T} \exp \left[-i \int_{0}^{T} d t \hat{H}_{\mathcal{S}}(t)\right]$. The JE (1) is fulfilled for an arbitrary $\mathrm{U}$ if the work assigned to given outcomes $a$ and $b$ is defined by $W(a, b)=E_{b}-E_{a}$ (see Appendix A).

Corrections and alternative formulations of the JE have been proposed for nonunitary evolution, including decoherence, heat exchange, and intermediate measurements [4,23,33-42], which we do not consider here.

While the classical JE and its TPM quantum version are formally identical, there is an important difference from the operational point of view. The classical work values can be determined by measuring an externally applied force along a path. Such a tool is by construction missing in the TPM scheme. This issue is closely related to the fact that "work is not an observable" [22] on the system. $W=W(a, b)$ is not given by a quantum expectation value but is assigned to a sequence of outcomes $\{a, b\}$ [29].

In the following we provide a framework which allows one to define work as an external quantum observable on a control system, similar in spirit to the measurement of work in a classical setting. This approach allows us to measure work independently of the knowledge of the system Hamiltonian. In the second part we look at the conceptual discrepancy between TPM schemes and classical work measurements for the determination of free energy differences and motivate how our proposal could overcome these issues.

Finally, we construct an operational work fluctuation theorem for the free energy difference which shows that quantum coherences can prevent us from finding the optimal $\Delta F$. We illustrate this with two examples and simulate the 
variational estimation of free energy differences for unknown Hamiltonians.

\section{RELATIVE HAMILTONIANS}

As in the classical experiments we separate the system of interest $\mathcal{S}$ (e.g., the RNA) from the control device $\mathcal{C}$ (e.g., the AFM). Usually, varying quantum Hamiltonians are modeled by an operator $\hat{H}_{\mathcal{S}}(\lambda)$, where $\lambda$ is a parameter associated with an external classical system, e.g., a varying magnetic field. In such an approach the classical part, by construction, cannot be treated quantum mechanically. Therefore, to derive an operationally accessible description of an external force which results in a change of the system Hamiltonian we need an ansatz which includes the external device in the formalism of quantum mechanics, that is, the global dynamics should be modeled by unitary evolution and quantum measurements.

We consider a system $\mathcal{S}$ coupled to a control device $\mathcal{C}$ by a Hamiltonian $\hat{H}_{\mathcal{S C}}$. In general the interaction will create entanglement between $\mathcal{S}$ and $\mathcal{C}$. However, we expect the control system not to be entangled but in an objective pure state, otherwise it would not be justified to consider it to be in a definite state representing a parameter of the system Hamiltonian. To keep $\mathcal{C}$ in a well-defined state we reset it rapidly to a definite state $\left|\psi_{\mathcal{C}}\right\rangle$. In a Zeno-like limit (fast resetting) $\mathcal{S}$ does not entangle anymore with $\mathcal{C}$. We model the resetting by a sequence of ancilla control systems $\mathcal{C}_{i}$ all prepared in the same initial state $\left|\psi_{\mathcal{C}}\right\rangle$ [43]. Each ancilla interacts once with $\mathcal{S}$ by the interaction $\hat{H}_{\mathcal{S C}}$. We assume the coupling time $\Delta t$ to be short such that the single-collision map for the system state $\rho_{\mathcal{S}}$ is given by

$$
\begin{aligned}
\rho_{\mathcal{S}}^{\prime} & =\operatorname{Tr}_{\mathcal{C}}\left\{e^{-i \hat{H}_{\mathcal{S C}} \Delta t}\left(\rho_{\mathcal{S}} \otimes\left|\psi_{\mathcal{C}}\right\rangle\left\langle\psi_{\mathcal{C}}\right|\right) e^{i \hat{H}_{\mathcal{S C}} \Delta t}\right\} \\
& =\rho_{\mathcal{S}}-i \Delta t\left[\left\langle\psi_{\mathcal{C}}\left|\hat{H}_{\mathcal{S C}}\right| \psi_{\mathcal{C}}\right\rangle, \rho_{\mathcal{S}}\right]+O\left(\Delta t^{2}\right) .
\end{aligned}
$$

In a continuous limit $(\Delta t \rightarrow d t)$ we obtain an effective unitary von Neumann dynamics in $\mathcal{S}$ [44],

$$
\dot{\rho}_{\mathcal{S}}=-i\left[\left\langle\psi_{\mathcal{C}}\left|\hat{H}_{\mathcal{S C}}\right| \psi_{\mathcal{C}}\right\rangle, \rho_{\mathcal{S}}\right]=-i\left[\hat{H}_{\mathcal{S}}^{\left|\psi_{\mathcal{C}}\right\rangle}, \rho_{\mathcal{S}}\right],
$$

given by the effective Hamiltonian $\hat{H}_{\mathcal{S}}^{\left|\psi_{\mathcal{C}}\right\rangle}=\left\langle\psi_{\mathcal{C}}\left|\hat{H}_{\mathcal{S C}}\right| \psi_{\mathcal{C}}\right\rangle$, which we denote the relative Hamiltonian on $\mathcal{S}$ with respect to the control state $\left|\psi_{\mathcal{C}}\right\rangle$. The analogy to the classical case is obvious. There the potential in the Hamiltonian can depend on the setting of an external control parameter, e.g., the displacement of a spring pulling the system. The Zeno-like resetting applies to the control device only. The system is not measured. As shown in Ref. [45] this would trivialize the work statistics.

A change of the local Hamiltonian in $\mathcal{S}$ can now be implemented by changing the initial states $\left|\psi_{\mathcal{C}}(t)\right\rangle$ of the ancillas (before their collision), which results in a time-dependent Hamiltonian $\hat{H}_{\mathcal{S}}^{\left|\psi_{\mathcal{C}}(t)\right\rangle}$.

\section{WORK}

Within this collision model framework we can define work operationally as an external observable. Varying $\hat{H}_{\mathcal{S}}^{\left|\psi_{\mathcal{C}}\right\rangle}$ leads to a change in the internal energy of $\mathcal{S}$ which can be seen as work performed on the system by the external control. We assume $\left|\psi_{\mathcal{C}}(t)\right\rangle$ to be differentiable. Between two collisions the experimenter changes the control system $\left|\psi_{\mathcal{C}}\right\rangle \mapsto$
$\left|\psi_{\mathcal{C}}^{\prime}\right\rangle=\left|\psi_{\mathcal{C}}\right\rangle+\left|\dot{\psi}_{\mathcal{C}}\right\rangle d t$. This results in a change of the effective Hamiltonian on the system $\hat{H}_{\mathcal{S}}^{\left|\psi_{\mathcal{C}}\right\rangle} \mapsto \hat{H}_{\mathcal{S}}^{\left|\psi_{\mathcal{C}}^{\prime}\right\rangle}$. Accordingly, the energy expectation value of $\mathcal{S}$ in a state $\left|\psi_{\mathcal{S}}\right\rangle$ changes to first order in $d t$ as

$$
\begin{aligned}
d W & =\left\langle\psi_{\mathcal{C}}^{\prime}\left|\left\langle\psi_{\mathcal{S}}\left|\hat{H}_{\mathcal{S C}}\right| \psi_{\mathcal{S}}\right\rangle\right| \psi_{\mathcal{C}}^{\prime}\right\rangle-\left\langle\psi_{\mathcal{C}}\left|\left\langle\psi_{\mathcal{S}}\left|\hat{H}_{\mathcal{S C}}\right| \psi_{\mathcal{S}}\right\rangle\right| \psi_{\mathcal{C}}\right\rangle \\
& =\left\langle\psi_{\mathcal{C}}^{\prime}\left|\hat{H}_{\mathcal{C}}^{\left|\psi_{\mathcal{S}}\right\rangle}\right| \psi_{\mathcal{C}}^{\prime}\right\rangle-\left\langle\psi_{\mathcal{C}}\left|\hat{H}_{\mathcal{C}}^{\left|\psi_{\mathcal{S}}\right\rangle}\right| \psi_{\mathcal{C}}\right\rangle \\
& =d t\left\langle\dot{\psi}_{\mathcal{C}}\left|\hat{H}_{\mathcal{C}}^{\left|\psi_{\mathcal{S}}\right\rangle}\right| \psi_{\mathcal{C}}\right\rangle+\left\langle\psi_{\mathcal{C}}\left|\hat{H}_{\mathcal{C}}^{\left|\psi_{\mathcal{S}}\right\rangle}\right| \dot{\psi}_{\mathcal{C}}\right\rangle d t+O\left(d t^{2}\right) \\
& =i d t\left\langle\dot{\psi}_{\mathcal{C}} \mid \psi_{\mathcal{C}}^{\xi}\right\rangle-i d t\left\langle\psi_{\mathcal{C}}^{\xi} \mid \dot{\psi}_{\mathcal{C}}\right\rangle \\
& =-2 d t \operatorname{Im}\left\{\left\langle\dot{\psi}_{\mathcal{C}} \mid \psi_{\mathcal{C}}^{\xi}\right\rangle\right\}
\end{aligned}
$$

where we have used that the state of a single ancilla after its collision with $\mathcal{S}$ in state $\left|\psi_{\mathcal{S}}\right\rangle$ is given by $\left|\psi_{\mathcal{C}}^{*}\right\rangle=$ $\left|\psi_{\mathcal{C}}\right\rangle-i \hat{H}_{\mathcal{C}}^{\left|\psi_{\mathcal{S}}\right\rangle}\left|\psi_{\mathcal{C}}\right\rangle d t=\left|\psi_{\mathcal{C}}\right\rangle+\left|\psi_{\mathcal{C}}^{\xi}\right\rangle d t$ (see Appendix B). The work increment $d W$ on $\mathcal{S}$ is encoded in the change of the ancilla's initial state $\left|\dot{\psi}_{\mathcal{C}}\right\rangle$ (controlled by the experimenter) and the change of the ancilla state due to an interaction with the system $\left|\psi_{\mathcal{C}}^{\xi}\right\rangle$ (can be measured by the experimenter). Crucially, knowledge about the Hamiltonian $\hat{H}_{\mathcal{S C}}$ is not needed.

To determine $d W$, an observable on the state of the ancilla $\left|\psi_{\mathcal{C}}^{\star}\right\rangle$ after its collision with $\mathcal{S}$ has to be measured. This quantum measurement yields, in general, random outcomes. Thus, we are looking for an observable on $\mathcal{C}$ whose expectation value is the performed $d W$. We can build the work increment observable for a single step in the collision model with the following orthogonal vectors which contain only states that are known to the experimenter:

$$
\left|\phi_{+}\right\rangle=\left|\psi_{\mathcal{C}}\right\rangle+i \alpha\left|\dot{\psi}_{\mathcal{C}}\right\rangle, \quad\left|\phi_{-}\right\rangle=\left|\psi_{\mathcal{C}}\right\rangle-i \alpha\left|\dot{\psi}_{\mathcal{C}}\right\rangle,
$$

with $\alpha=\sqrt{\left\langle\psi_{\mathcal{C}} \mid \psi_{\mathcal{C}}\right\rangle /\left\langle\dot{\psi}_{\mathcal{C}} \mid \dot{\psi}_{\mathcal{C}}\right\rangle}$. The work increment observable reads (see Appendix C)

$$
\begin{aligned}
\Omega & =\frac{1}{2 \alpha}\left(\left|\phi_{-}\right\rangle\left\langle\phi_{-}|-| \phi_{+}\right\rangle\left\langle\phi_{+}\right|\right)+\zeta \mathbb{1} \\
& =i\left(\left|\psi_{\mathcal{C}}\right\rangle\left\langle\dot{\psi}_{\mathcal{C}}|-| \dot{\psi}_{\mathcal{C}}\right\rangle\left\langle\psi_{\mathcal{C}}\right|\right)+\zeta \mathbb{1},
\end{aligned}
$$

with $\zeta=2 \operatorname{Im}\left\{\left\langle\dot{\psi}_{\mathcal{C}} \mid \psi_{\mathcal{C}}\right\rangle\right\}$ known to the experimenter. The observable $\Omega$ yields the correct average work increment (to the first order in $d t$ ) for the single step $\left\langle\psi_{\mathcal{C}}^{\star}|\Omega| \psi_{\mathcal{C}}^{\star}\right\rangle=d W$. Concatenating the work increments $d W$, we are able to operationally determine work performed on a system $\mathcal{S}$ by measuring a control system $\mathcal{C}$ without knowing the Hamiltonians. This is in strong analogy to classical pulling experiments, where work is determined by measurements of an external force along a path of the control system.

Applying the collision model driving to a system starting in $\rho_{\mathcal{S}}$, leads to a unitary evolution $\rho_{\mathcal{S}}^{\prime}=\mathrm{U} \rho_{\mathcal{S}} \mathrm{U}^{\dagger}$. The work supplied by $\mathcal{C}$ has to be measured over many runs with the same initial state and the same protocol $\left|\psi_{\mathcal{C}}(t)\right\rangle$, since the quantum observables $\Omega$ yield $d W$ as their expectation values [46]. The total $\langle W\rangle$ along the protocol is the change of the energy expectation value during the evolution $\langle W\rangle=$ $\operatorname{Tr}\left\{\hat{H}_{\mathcal{S}}^{B} \rho_{\mathcal{S}}^{\prime}\right\}-\operatorname{Tr}\left\{\hat{H}_{\mathcal{S}}^{A} \rho_{\mathcal{S}}\right\}$. Starting in a mixed state $\rho_{\mathcal{S}}$, the observable will give only the average change of energy. This is crucial for the development of a JE as we will see later. 


\section{ON THE PRACTICAL RELEVANCE OF TPM-JES}

The TPM scheme has been proven to be a useful tool for the theoretical description of nonequilibrium thermodynamics, in particular for JEs. Quantum features like coherences and measurement backactions have been successfully addressed in this and similar frameworks [39,47-54]. However, despite the formal agreement with classical JEs, it is questionable whether TPM-JEs are of the same practical relevance as the classical ones, since the latter obtain the work distribution in a very different manner. Before we continue, we briefly comment on that issue and motivate how our framework could close this conceptual gap.

In a TPM-JE experiment two projective energy measurements are performed. Knowing which energies $E_{k}$ have to be assigned to the outcomes of these measurements and knowing that the outcomes correspond to distinct eigenstates $|k\rangle$ is equivalent to knowing the Hamiltonian $\hat{H}$ which corresponds to such an energy measurement. This, however, allows one to calculate $\Delta F$ for the two Hamiltonians $\hat{H}_{A / B}$ directly from their partition functions $Z_{A / B}$. In other words, both sides of a quantum TPM-JE would be determined from the same inputs, namely, $\hat{H}_{A / B}$ [55]. A TPM-JE which explicitly assumes and describes how the work could be measured without knowledge of the Hamiltonians is - to the best of our knowledge-missing in the literature.

It is insightful to consider a TPM scenario in the classical RNA experiment. At time $t=0$ the experimenter determines the microstate of the molecule, i.e., she measures position and momentum of each atom (in principle possible in a classical experiment). Then, she plugs this phase space state into the (classical) Hamiltonian of the whole molecule, which she needs to have at hand to determine the energy of the particular microstate in this run. After applying the driving protocol she measures the new microstate and plugs it into the final Hamiltonian. This classical TPM scheme could in principle be implemented and it would verify the JE, but it would hardly be considered as a breakthrough for the experimental accessibility of equilibrium quantities through nonequilibrium processes. We emphasize that this is not an insufficiency of the possible experimental realizations, such as finite number of runs, noise, energy stored in the control system, or problems with convergence $[6,56-60]$ but a conceptual issue of the TPM scheme.

Alternative approaches avoiding the TPM scenario have been proposed and experimentally implemented. They rely, e.g., on interferometric, ancilla-assisted schemes [61-67], or work reservoirs $[39,47]$ or use quantum trajectory techniques [35,43,68-74]. These frameworks have all shed new light on quantum thermodynamics. Although the knowledge of the initial and final Hamiltonians is still necessary in these scenarios, many of them could certainly be extended to the case of unknown Hamiltonians.

While our framework is conceptually close to the classical approach, we will see that quantumness hinders us from applying it straightforwardly to a JE. The work can be measured externally without knowing the Hamiltonian, but since $\Omega$ is a quantum observable, only its expectation value is a meaningful quantity. Thus, starting in a thermal state, the approach can measure only the average work $\langle W\rangle$. Nevertheless, while in the TPM scheme both $\hat{H}_{\mathcal{S}}^{A}$ and $\hat{H}_{\mathcal{S}}^{B}$ are known, the externally observed work allows to construct a one-point measurement scheme which can determine $\Delta F$ in an operationally meaningful way.

\section{OPERATIONAL QUANTUM ONE-POINT MEASUREMENT JE}

To obtain a work distribution which satisfies a JE, the experimenter needs to prepare an objective ensemble of energy eigenstates representing the initial thermal state. She can do so by measuring the initial state in the basis of $\hat{H}_{\mathcal{S}}^{A}$, which means that also in this scenario that basis has to be known. However, from the operational point of view, there is a difference between $\hat{H}_{\mathcal{S}}^{A}$ and $\hat{H}_{\mathcal{S}}^{B}$ in a quantum JE experiment. While the initial Hamiltonian can be operationally determined by state tomography, the final one could be obtained only by process tomography since the system never thermalizes in $\hat{H}_{\mathcal{S}}^{B}$.

We propose the following operational one-point measurement scheme. In each run, the system starts in a thermal state. At $t=0$ the system is measured in the energy eigenbasis with outcome $a$ and the work is obtained by measuring the observables $\Omega$. After sufficiently many runs the work associated with outcome $a$ can be determined, and we get

$$
\left\langle W_{a}\right\rangle=\operatorname{Tr}\left\{\hat{H}_{\mathcal{S}}^{B} \mathrm{U}|a\rangle\langle a| \mathrm{U}^{\dagger}\right\}-\operatorname{Tr}\left\{\hat{H}_{\mathcal{S}}^{A}|a\rangle\langle a|\right\} .
$$

These work values do not satisfy the JE (1), but it has been shown in Refs. [75,76] that they fulfill a modified equality

$$
\mathbb{E}_{a}\left[e^{-\beta\left\langle W_{a}\right\rangle}\right]=e^{-\beta \Delta F} e^{-S\left(\tilde{\rho}_{T} \| \rho_{T}^{\text {th }}\right)}=e^{-\beta \Delta \tilde{F}},
$$

where $S\left(\tilde{\rho}_{T} \| \rho_{T}^{\text {th }}\right)$ is the quantum relative entropy between a fictitious "best guess" state (see Appendix D) and the thermal state of the final Hamiltonian. $\left\langle W_{a}\right\rangle$ is the quantum expectation value for the work performed whenever the initial measurement yields $a$, whereas $\mathbb{E}_{a}$ denotes the classical average over all outcomes $a$.

The right-hand side of Eq. (8) was originally derived to show the thermodynamic cost of the second measurement in a TPM scheme. It depends on the time evolution $U$ generated by the driving. Crucially, $\tilde{\rho}_{T}$ and $\rho_{T}^{\text {th }}$ remain unknown in our approach. Thus, Eq. (8) cannot directly be used to obtain $\Delta F$. However, the relative entropy $S\left(\tilde{\rho}_{T} \| \rho_{T}^{\text {th }}\right)$ is always nonnegative and vanishes only if $\tilde{\rho}_{T}=\rho_{T}^{\text {th }}$. Thus [75],

$$
\Delta F=\Delta \tilde{F}-\frac{1}{\beta} S\left(\tilde{\rho}_{T} \| \rho_{T}^{\text {th }}\right) \leqslant \Delta \tilde{F} \leqslant\langle W\rangle .
$$

Therefore, an optimization over possible protocols which start in $\hat{H}_{\mathcal{S}}^{A}$ and end in $\hat{H}_{\mathcal{S}}^{B}$ can provide tighter bounds on $\Delta F$ because the resulting unknown $U$ might lead to a lower relative entropy. The true $\Delta F$ is obtained for $S\left(\tilde{\rho}_{T} \| \rho_{T}^{\text {th }}\right)=0$, which is the case whenever $\left[\mathrm{U}^{\dagger} \hat{H}_{\mathcal{S}}^{B} \mathrm{U}, \hat{H}_{\mathcal{S}}^{A}\right]=0$. Therefore, in principle, $\Delta F$ is operationally obtainable without knowledge of $\hat{H}_{\mathcal{S}}^{B}$.

\section{EXAMPLES}

\section{A. Qubit system, qubit control}

In a first example, $\mathcal{S}$ and $\mathcal{C}$ are qubits. The change of the Hamiltonian in $\mathcal{S}$ is given by a sequence of ancilla qubits 


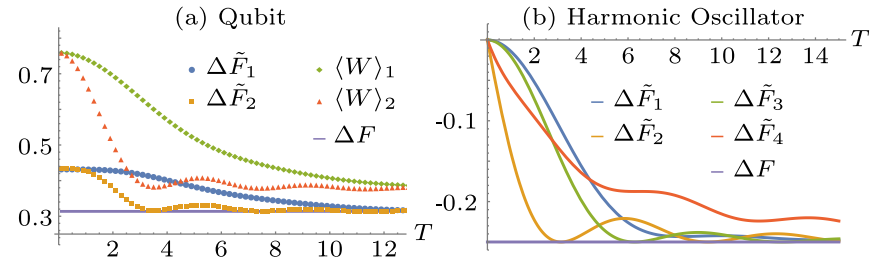

FIG. 1. (a) Qubit system and qubit control: We plot the correct $\Delta F$ (nonoperational), the measurable $\Delta \tilde{F}_{1 / 2}$, and the average work $\langle W\rangle_{1 / 2}$ for different interaction Hamiltonians $\hat{H}_{\mathcal{S C}}^{1 / 2}$ and protocols (see main text) in dependence of the unitless switching time $\mathrm{T}$. The protocols are approximated by 40000 steps and $\beta=1$. (b) Harmonic oscillator system and qubit control: We plot the inaccessible correct $\Delta F$ and the measurable $\Delta \tilde{F}$ for four different forcing protocols with a fixed $\hat{H}_{\mathcal{S C}}$. Depending on the shape of the driving function and the imaginary part of the force, one can reach $\Delta \tilde{F}=\Delta F$ not only in the quantum adiabatic limit but also in finite time. We set $\beta=\omega=g=1$.

$\mathcal{C}_{i}$ initialized in states $\left|\psi_{\mathcal{C}}\left(t_{i}\right)\right\rangle$ with coupling $\hat{H}_{\mathcal{S C}}^{1}=\sigma_{x} \otimes$ $|0\rangle\left\langle 0\left|-(1 / 2) \sigma_{y} \otimes\right| 1\right\rangle\langle 1|$. The $\left|\psi_{\mathcal{C}}\left(t_{i}\right)\right\rangle$ 's are parametrized by two functions $\theta(t)$ and $\phi(t)$ with $\left|\psi_{\mathcal{C}}\left(t_{i}\right)\right\rangle=\cos \left[\theta\left(t_{i}\right) / 2\right]|0\rangle+$ $\exp \left[i \phi\left(t_{i}\right)\right] \sin \left[\theta\left(t_{i}\right) / 2\right]|1\rangle$. The relative Hamiltonian on $\mathcal{S}$ depends only on $\theta$ :

$$
\hat{H}_{\mathcal{S}}^{1}(\theta)=\cos ^{2} \frac{\theta\left(t_{i}\right)}{2} \sigma_{x}-\frac{1}{2} \sin ^{2} \frac{\theta\left(t_{i}\right)}{2} \sigma_{y} .
$$

Starting the protocol at $\theta(0)=0$ and ending with $\theta(T)=\pi$ leads to $\Delta F=-(1 / \beta) \ln [\cosh (\beta) / \cosh (\beta / 2)]$. In Fig. 1(a) we plot $\Delta F, \Delta \tilde{F}_{1}$ and $\langle W\rangle_{1}$ for different switching times $T$. The switching function is $\theta(t)=(\pi / T) t$, and we approximate the protocol by $N=40000$ collisions each of length $\Delta t=T / N$. In the limit of short switching times $T$ the reduced state stays diagonal in $\hat{H}_{\mathcal{S}}^{A}$, and, since $\left[\hat{H}_{\mathcal{S}}^{A}, \hat{H}_{\mathcal{S}}^{B}\right] \neq 0$, the modified JE (9) does not give the correct $\Delta F$ because of the relative entropy contribution. Nevertheless, the value is better than the bound given by $\langle W\rangle_{1}$. For large $T$ we are in the regime of quantum adiabatic evolution (not to be confused with thermodynamic adiabaticity). The system approximately stays diagonal in the eigenbasis of the instantaneous Hamiltonian during the whole protocol. Thus, $S\left(\tilde{\rho}_{T} \| \rho_{T}^{\text {th }}\right)=0$ and $\Delta \tilde{F}_{1}=\Delta F$.

Taking another interaction Hamiltonian $\hat{H}_{\mathcal{S C}}^{2}=2\left(\sigma_{+} \otimes\right.$ $\left.\sigma_{-}+\sigma_{-} \otimes \sigma_{+}\right)$we get a dependence on both $\theta$ and $\phi$ :

$$
\hat{H}_{\mathcal{S}}^{2}(\theta, \phi)=e^{i \phi} \sin (\theta) \sigma_{+}+e^{-i \phi} \sin (\theta) \sigma_{-} .
$$

We set $\theta(0)=\pi / 2, \theta(T)=\pi / 6, \phi(0)=0$, and $\phi(T)=\pi / 2$, which leads to the same $\hat{H}_{A / B}$ and, therefore, to the same $\Delta F$ as in the example above. The functions $\theta(t)$ and $\phi(t)$ are linear. In Fig. 1(a) we see that $\Delta \tilde{F}_{2}$ and $\langle W\rangle_{2}$ show a different behavior under this protocol and the correct $\Delta F$ can be reached in finite time where the evolution cannot be considered approximately quantum adiabatic.

\section{B. Displaced harmonic oscillator}

To illustrate the relevance of the entropic contribution $S\left(\tilde{\rho}_{T} \| \rho_{T}^{\text {th }}\right)$ we consider a harmonic oscillator system $\mathcal{S}$. The control $\mathcal{C}$ is again a qubit. The interaction is $\hat{H}_{\mathcal{S C}}=\omega\left(a^{\dagger} a+\right.$ $1 / 2)+g\left(a \otimes \sigma_{+}+a^{\dagger} \otimes \sigma_{-}\right)$, where $g$ is a real coupling constant. The relative Hamiltonian reads

$$
\hat{H}_{\mathcal{S}}(\theta, \phi)=\omega\left(a^{\dagger} a+\frac{1}{2}\right)+\frac{g}{2} \sin (\theta)\left(e^{i \phi} a^{\dagger}+e^{-i \phi} a\right),
$$

which describes a harmonic oscillator displaced by a complex force. The free energy is independent of $\phi$, and we get $\Delta F=$ $-\left[g^{2} /(4 \omega)\right]\left[\sin ^{2}\left(\theta_{B}\right)-\sin ^{2}\left(\theta_{A}\right)\right]$.

The work performed on $\mathcal{S}$ is independent of the initial energy eigenstate [77]. Therefore, the initial energy measurement is superfluous and $\Delta \tilde{F}=\langle W\rangle$. In a classical system this would be rather boring. In the quantum case, however, it allows one to directly study the contribution of the relative entropy by measuring the average work externally. In Fig. 1(b) we show the measurable $\Delta \tilde{F}$ for different protocols $\theta(t)$ and $\phi(t)$ with the same $\hat{H}_{A / B}$ for different switching times $T$ in a continuous limit. Details about the different protocols may be found in Appendix E. Most importantly, the correct $\Delta F$ can be determined not only in the quantum adiabatic limit but also at finite times if the switching protocol is suitably chosen.

\section{SIMULATIONS FOR UNKNOWN HAMILTONIANS}

The modified JE in Eq. (8) can provide only an upper bound $\Delta \tilde{F}$ for the true free energy difference $\Delta F$. In an experiment one can try to minimize $\Delta \tilde{F}$ by varying the protocol, i.e., the function $\left|\psi_{\mathcal{C}}(t)\right\rangle$. Such a variational approach cannot guarantee to find the global minimum $\Delta F$ for a finite duration of the protocol. However, especially for small quantum systems, even a very basic optimization strategy can provide reasonable values $\Delta \tilde{F}_{\text {opt }}$ close to $\Delta F$.

To show that the examples above are not just fine-tuned cases, we simulate the free energy difference estimation for random Hamiltonians $\hat{H}_{\mathcal{S C}}$. The control system $\mathcal{C}$ is again a qubit. For the system of interest $\mathcal{S}$ we choose finite quantum systems of different dimension $d \in\{2,3,4,5,6\}$. The control state is again parametrized by two functions $\theta(t)$ and $\phi(t)$ :

$$
\left|\psi_{\mathcal{C}}(t)\right\rangle=\cos [\theta(t) / 2]|0\rangle+\exp [i \phi(t)] \sin [\theta(t) / 2]|1\rangle .
$$

The initial and final values are always given by $\theta(0)=0$, $\theta(T)=\pi / 2$ and $\phi(0)=\phi(T)=0$, fixing the initial and final relative Hamiltonians on $\mathcal{S}$ for a given $\hat{H}_{\mathcal{S C}}$.

The aim is now to find functions $\theta(t)$ and $\phi(t)$ which minimize $\Delta \tilde{F}$. We constrain the total duration $T$ of the protocol to an interval $T \in\left[T_{\min }, T_{\max }\right]$ to ensure a finite-time protocol and avoid the quantum adiabatic regime $T \rightarrow \infty$.

We combine an optimization of the duration $T$ and a gradient descent approach for the optimization of the shapes of $\theta(t)$ and $\phi(t)$ :

(1) A start protocol given by a linear function $\theta(t)=$ $(\pi / T) t$ and a constant function $\phi(t)=0$ is applied for different $T \in\left[T_{\min }, T_{\max }\right]$. The duration $T_{\text {opt }}$ which yields the minimum $\Delta \tilde{F}$ is then used in the next step.

(2) For the fixed $T_{\text {opt }}$, the functions $\theta(t)$ and $\phi(t)$ are now optimized to further reduce $\Delta \tilde{F}$. For this purpose we model $\theta$ and $\phi$ as a spline interpolation of $n$ equally spaced sampling points between the start $(t=0)$ and the end $\left(t=T_{\text {opt }}\right)$ of the protocol. Thus, the functions are parametrized by two vectors $\vec{s}_{\theta}$ and $\vec{s}_{\phi}$ with $n$ entries. The optimization is then done by a 

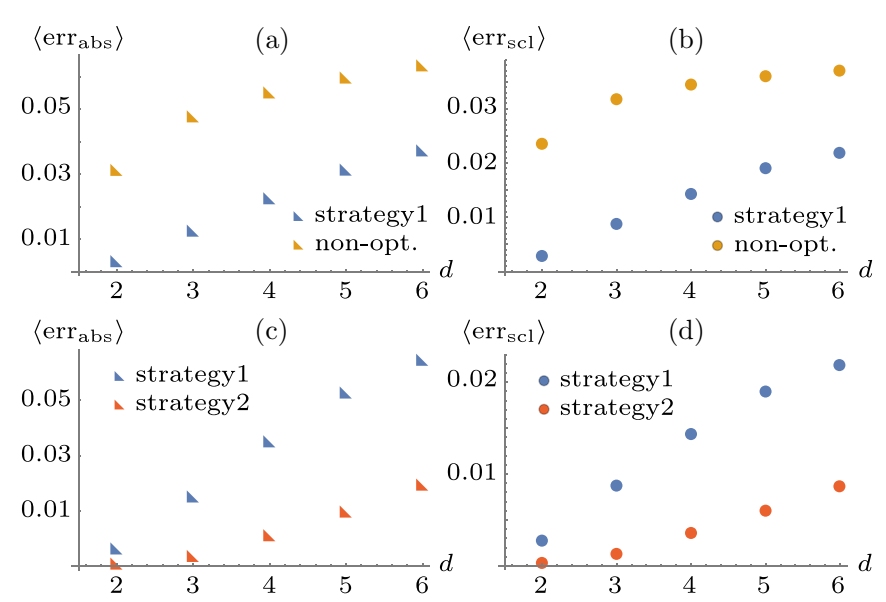

FIG. 2. (a) The average errors are plotted for different system dimensions $d$. The blue triangles were obtained with the optimization parameters $T_{\max }=5$ and $n=5$. The orange triangles show the average errors for the nonoptimized linear start protocol and $T=1$. (b) The average scaled errors are plotted. The curves show that the variational approach can optimize $\Delta \tilde{F}$ for different (unknown) strengths of the underlying Hamiltonian, always with the same strategy. (c), (d) The average errors and the average scaled errors are shown for the two sets of optimization parameters. Strategy 2 ( $T_{\max }=20$ and $n=10$ ) significantly improves the results.

gradient descent approach for $\vec{s}_{\theta}$ and $\vec{s}_{\phi}$, initialized with the spline representation of the linear start protocol.

(3) Between the steps of the gradient descent method the duration $T$ is again optimized and a new $T_{\mathrm{opt}}$ is set.

For the first simulations we choose the following values:

$$
T_{\min }=0.5, \quad T_{\max }=5, \quad n=5 .
$$

The Hamiltonians are randomly sampled with bounded eigenvalues between -1 and 1 . For each dimension $d$ of the system we have $N_{H}=1500$ Hamiltonians. To see how well the $\Delta \tilde{F}_{\text {opt }}$ found by the optimization strategy approximates the correct $\Delta F$ we calculate the errors

$$
\operatorname{err}_{\mathrm{abs}}=\left|\Delta F-\Delta \tilde{F}_{\mathrm{opt}}\right|
$$

for each sampled Hamiltonian $(\beta=1)$ and take the average $\left\langle\operatorname{err}_{\mathrm{abs}}\right\rangle$. The results are plotted in Fig. 2(a). For comparison, we also show the average error for the nonoptimized linear start protocol of duration $T=1$.

To get an impression how well the approximation works for Hamiltonians of different strengths, we also calculate scaled errors,

$$
\operatorname{err}_{\mathrm{scl}}=\left|\frac{\Delta F-\Delta \tilde{F}_{\mathrm{opt}}}{\Delta \lambda}\right|,
$$

where $\Delta \lambda=\lambda_{\max }-\lambda_{\min }$ is the spread between the largest and smallest eigenvalue of the sampled underlying Hamiltonian $\hat{H}_{\mathcal{S C}}$ and can be seen as the relevant global energy scale. In Fig. 2(b) we plot the average over the scaled errors, showing that the method yields good approximations independently of the energy scale. The optimization strategy significantly decreases the errors for any dimension considered. Thus, one can generally expect to find a better bound by optimizing the protocol. For the qubit case the strategy yields values close to the exact ones. As expected, for higher dimensions the deviations increase.

By slightly improving the optimization parameters, we obtain reasonably well approximated bounds also for higher dimensions. We set $T_{\max }=20$ and increase the number of points for the spline approximation to $n=10$. Figures 2(c) and 2(d) show that now the average errors for systems of $d=6$ are decreased to a value which can be obtained for qutrit systems under strategy 1 .

All in all, these basic strategies demonstrate that the variational approach can indeed provide reasonable values $\Delta \tilde{F} \approx$ $\Delta F$. More sophisticated optimization methods could surely improve these results and render the modified JE suitable also for even higher dimensions of the system.

\section{CONCLUSION}

We address the question how to construct a quantum observable for externally measuring work in analogy to the classical case of a forced system. In our framework, the control system is included in the quantum domain. Since the control parameter for the system Hamiltonian is assumed to be well defined, the control system needs to be in a well-defined quantum state, known to the experimenter, at all times. We implement this by a repeated measure-and-prepare approach in a Zeno-like limit with a suitable work increment observable.

We argue that the practical relevance of standard definitions of quantum work for the estimation of free energy differences is questionable if the approaches depend on the knowledge of the initial and final Hamiltonian. Using our framework, we construct an operational measurement scheme which satisfies a modified JE and is able to extend quantum work fluctuation theorems to unknown Hamiltonians.

We illustrate by relevant examples that the model is suitable for the operational determination of bounds on free energy differences. In contrast to the classical case, a quantum contribution, stemming from coherences in the final system state, prevents us, in general, from obtaining the correct $\Delta F$ directly. This contribution vanishes in a quantum adiabatic limit but can also be avoided by a suitable variational optimization of the driving protocol.

An experimental implementation would in principle allow to obtain the left- and right-hand side of a quantum JE independently of each other. The one side by an external measurement of different work trajectories (e.g., in a quantum adiabatic regime), the other side of the equation by a thermodynamic reversible process including a heat bath and measuring the average work.

Our approach could be applied to more general quantum fluctuation theorems. As the quantum contribution in the modified JE shows, the focus on operationally accessible quantities can help to understand better the fundamental differences between quantum and classical fluctuation relations.

\section{ACKNOWLEDGMENT}

The authors would like to thank Dario Egloff for helpful discussions. 


\section{APPENDIX A: QUANTUM JE IN A TPM SCHEME}

As in the classical case, one assumes the system to be initially in a thermal state of Hamiltonian $\hat{H}_{A}$,

$$
\rho_{\mathcal{S}}=\frac{1}{Z_{A}} \sum_{a} e^{-\beta E_{a}}|a\rangle\langle a|, \quad Z_{A}=\sum_{a} e^{-\beta E_{a}} .
$$

Therefore, the probability to measure outcome $a$ and $b$ in first and second measurement, respectively, is then given by

$$
p(a, b)=\operatorname{Tr}\left\{|b\rangle\langle b|\mathrm{U}| a\rangle\left\langle a\left|\rho_{\mathcal{S}}\right| a\right\rangle\langle a| \mathrm{U}^{\dagger}\right\},
$$

where we have assumed that the first measurement is implemented by a Lüders instrument [78]. The classical form of the JE can then directly be verified for the quantum TPM scheme $[79,80]$

$$
\begin{aligned}
\left\langle e^{-\beta W}\right\rangle & =\sum_{a, b} p(a, b) e^{-\beta\left(E_{b}-E_{a}\right)} \\
& =\sum_{a, b} \operatorname{Tr}\left\{\mathbb{Q}_{b} \mathrm{U} \mathbb{P}_{a} \rho_{\mathcal{S}} \mathbb{P}_{a} \mathrm{U}^{\dagger}\right\} e^{-\beta\left(E_{b}-E_{a}\right)} \\
& =\sum_{b} e^{-\beta E_{b}} \operatorname{Tr}\left\{\mathbb{Q}_{b} \mathrm{U} \sum_{a}\left(e^{+\beta E_{a}} \mathbb{P}_{a} \rho_{\mathcal{S}} \mathbb{P}_{a}\right) \mathrm{U}^{\dagger}\right\} \\
& =\sum_{b} e^{-\beta E_{b}} \operatorname{Tr}\left\{\mathbb{Q}_{b} \mathrm{U} \frac{1}{Z_{A}} \mathbb{1} \mathrm{U}^{\dagger}\right\} \\
& =\frac{1}{Z_{A}} \sum_{b} e^{-\beta E_{b}}=\frac{Z_{B}}{Z_{A}}=e^{-\beta \Delta F},
\end{aligned}
$$

where $\mathbb{P}_{a}=|a\rangle\langle a|$ and $\mathbb{Q}_{b}=|b\rangle\langle b|$. As one can see from the fourth line, the TPM-JE in this form works for any unital dynamics and is not restricted to unitary ones [51,81,82].

\section{APPENDIX B: EQUIVALENCE TO THE CHANGE OF THE INTERNAL ENERGY}

The work increment $d W$ for a single collision defined in Eq. (4) is (to first order in $d t$ ) equivalent to the standard definition for the change of the internal energy $d E$ in a closed quantum system. For a general quantum system we have [83]

$$
d E=\operatorname{Tr}\left\{\dot{\hat{H}}_{\mathcal{S}} \rho_{\mathcal{S}}\right\} d t+\operatorname{Tr}\left\{\hat{H}_{\mathcal{S}} \dot{\rho}_{\mathcal{S}}\right\} d t .
$$

The effective system dynamics is unitary and governed by the von Neumann equation for the relative Hamiltonian $\hat{H}_{\mathcal{S}}=\hat{H}_{\mathcal{S}}^{\left|\psi_{\mathcal{C}}\right\rangle}=\left\langle\psi_{\mathcal{C}}(t)\left|\hat{H}_{\mathcal{S C}}\right| \psi_{\mathcal{C}}(t)\right\rangle$ :

$$
\dot{\rho}_{\mathcal{S}}(t)=-i\left[\left\langle\psi_{\mathcal{C}}(t)\left|\hat{H}_{\mathcal{S C}}\right| \psi_{\mathcal{C}}(t)\right\rangle, \rho_{\mathcal{S}}\right]=-i\left[\hat{H}_{\mathcal{S}}^{\left|\psi_{\mathcal{C}}\right\rangle}, \rho_{\mathcal{S}}\right] .
$$

Therefore, the second term in Eq. (B1) vanishes because of the cyclic property of the trace. Assuming a pure system state $\left|\psi_{\mathcal{S}}(t)\right\rangle$ as in the main text, the first term reads

$$
\begin{aligned}
d E= & \left\langle\psi_{\mathcal{S}}(t)\left|\dot{\hat{H}}_{\mathcal{S}}(t)\right| \psi_{\mathcal{S}}(t)\right\rangle d t \\
= & \left\langle\psi_{\mathcal{S}}(t)\left|\left[\frac{\partial}{\partial t}\left\langle\psi_{\mathcal{C}}(t)\left|\hat{H}_{\mathcal{S C}}\right| \psi_{\mathcal{C}}(t)\right\rangle\right]\right| \psi_{\mathcal{S}}(t)\right\rangle d t \\
= & \left\langle\psi_{\mathcal{S}}(t)\left|\left\langle\dot{\psi}_{\mathcal{C}}(t)\left|\hat{H}_{\mathcal{S C}}\right| \psi_{\mathcal{C}}(t)\right\rangle\right| \psi_{\mathcal{S}}(t)\right\rangle d t \\
& +\left\langle\psi_{\mathcal{S}}(t)\left|\left\langle\psi_{\mathcal{C}}(t)\left|\hat{H}_{\mathcal{S C}}\right| \dot{\psi}_{\mathcal{C}}(t)\right\rangle\right| \psi_{\mathcal{S}}(t)\right\rangle d t
\end{aligned}
$$

$$
\begin{aligned}
= & \left\langle\dot{\psi}_{\mathcal{C}}(t)\left|\left\langle\psi_{\mathcal{S}}(t)\left|\hat{H}_{\mathcal{S C}}\right| \psi_{\mathcal{S}}(t)\right\rangle\right| \psi_{\mathcal{C}}(t)\right\rangle d t \\
& +\left\langle\psi_{\mathcal{C}}(t)\left|\left\langle\psi_{\mathcal{S}}(t)\left|\hat{H}_{\mathcal{S C}}\right| \psi_{\mathcal{S}}(t)\right\rangle\right| \dot{\psi}_{\mathcal{C}}(t)\right\rangle d t \\
= & \left\langle\dot{\psi}_{\mathcal{C}}(t)\left|\hat{H}_{\mathcal{C}}^{\left|\psi_{\mathcal{S}}(t)\right\rangle}\right| \psi_{\mathcal{C}}(t)\right\rangle d t \\
& +\left\langle\psi_{\mathcal{C}}(t)\left|\hat{H}_{\mathcal{C}}^{\left|\psi_{\mathcal{S}}(t)\right\rangle}\right| \dot{\psi}_{\mathcal{C}}(t)\right\rangle d t .
\end{aligned}
$$

The last line is to first order in $d t$ equivalent to the third line of Eq. (4).

\section{APPENDIX C: THE WORK INCREMENT OBSERVABLE}

The vectors needed to construct the work observable are

$$
\begin{aligned}
\left|\phi_{+}\right\rangle & =\left|\psi_{\mathcal{C}}\right\rangle+i \alpha\left|\dot{\psi}_{\mathcal{C}}\right\rangle, \quad\left|\phi_{-}\right\rangle=\left|\psi_{\mathcal{C}}\right\rangle-i \alpha\left|\dot{\psi}_{\mathcal{C}}\right\rangle, \\
\alpha & =\sqrt{\frac{\left\langle\psi_{\mathcal{C}} \mid \psi_{\mathcal{C}}\right\rangle}{\left\langle\dot{\psi}_{\mathcal{C}} \mid \dot{\psi}_{\mathcal{C}}\right\rangle}} .
\end{aligned}
$$

They are orthogonal and can therefore be used to define a suitable measurement on the ancillas after their collision

$$
\begin{aligned}
\left\langle\phi_{+} \mid \phi_{-}\right\rangle= & \left\langle\psi_{\mathcal{C}} \mid \psi_{\mathcal{C}}\right\rangle-\alpha^{2}\left\langle\dot{\psi}_{\mathcal{C}} \mid \dot{\psi}_{\mathcal{C}}\right\rangle \\
& -i \alpha\left\langle\dot{\psi}_{\mathcal{C}} \mid \psi_{\mathcal{C}}\right\rangle-i \alpha\left\langle\psi_{\mathcal{C}} \mid \dot{\psi}_{\mathcal{C}}\right\rangle \\
= & \left\langle\psi_{\mathcal{C}} \mid \psi_{\mathcal{C}}\right\rangle-\alpha^{2}\left\langle\dot{\psi}_{\mathcal{C}} \mid \dot{\psi}_{\mathcal{C}}\right\rangle \\
& -i \alpha\left\langle\dot{\psi}_{\mathcal{C}} \mid \psi_{\mathcal{C}}\right\rangle+i \alpha\left\langle\dot{\psi}_{\mathcal{C}} \mid \psi_{\mathcal{C}}\right\rangle \\
= & \left\langle\psi_{\mathcal{C}} \mid \psi_{\mathcal{C}}\right\rangle-\alpha^{2}\left\langle\dot{\psi}_{\mathcal{C}} \mid \dot{\psi}_{\mathcal{C}}\right\rangle \\
= & 0,
\end{aligned}
$$

where we have used that $\left\langle\dot{\psi}_{\mathcal{C}} \mid \psi_{\mathcal{C}}\right\rangle$ is purely imaginary. The observable reads

$$
\begin{aligned}
\Omega & =\frac{1}{2 \alpha}\left(\left|\phi_{-}\right\rangle\left\langle\phi_{-}|-| \phi_{+}\right\rangle\left\langle\phi_{+}\right|\right)+\zeta \mathbb{1} \\
& =i\left(\left|\psi_{\mathcal{C}}\right\rangle\left\langle\dot{\psi}_{\mathcal{C}}|-| \dot{\psi}_{\mathcal{C}}\right\rangle\left\langle\psi_{\mathcal{C}}\right|\right)+\zeta \mathbb{1},
\end{aligned}
$$

with

$$
\zeta=2 \operatorname{Im}\left\{\left\langle\dot{\psi}_{\mathcal{C}} \mid \psi_{\mathcal{C}}\right\rangle\right\}
$$

The expectation value of $\Omega$ is equal to the work increment

$$
\begin{aligned}
\left\langle\psi_{\mathcal{C}}^{\star}|\Omega| \psi_{\mathcal{C}}^{\star}\right\rangle= & i\left(\left\langle\psi_{\mathcal{C}} \mid \psi_{\mathcal{C}}\right\rangle\left\langle\dot{\psi}_{\mathcal{C}} \mid \psi_{\mathcal{C}}\right\rangle-\left\langle\psi_{\mathcal{C}} \mid \dot{\psi}_{\mathcal{C}}\right\rangle\left\langle\psi_{\mathcal{C}} \mid \psi_{\mathcal{C}}\right\rangle\right. \\
& +d t\left\langle\psi_{\mathcal{C}} \mid \psi_{\mathcal{C}}\right\rangle\left\langle\dot{\psi}_{\mathcal{C}} \mid \psi_{\mathcal{C}}^{\xi}\right\rangle-d t\left\langle\psi_{\mathcal{C}} \mid \dot{\psi}_{\mathcal{C}}\right\rangle\left\langle\psi_{\mathcal{C}} \mid \psi_{\mathcal{C}}^{\xi}\right\rangle \\
& +d t\left\langle\psi_{\mathcal{C}}^{\xi} \mid \psi_{\mathcal{C}}\right\rangle\left\langle\dot{\psi}_{\mathcal{C}} \mid \psi_{\mathcal{C}}\right\rangle \\
& \left.-d t\left\langle\psi_{\mathcal{C}}^{\xi} \mid \dot{\psi}_{\mathcal{C}}\right\rangle\left\langle\psi_{\mathcal{C}} \mid \psi_{\mathcal{C}}\right\rangle\right)+\zeta \\
= & -2 d t \operatorname{Im}\left\{\left\langle\dot{\psi}_{\mathcal{C}} \mid \psi_{\mathcal{C}}^{\xi}\right\rangle\right\}-2 \operatorname{Im}\left\{\left\langle\dot{\psi}_{\mathcal{C}} \mid \psi_{\mathcal{C}}\right\rangle\right\}+\zeta \\
= & -2 d t \operatorname{Im}\left\{\left\langle\dot{\psi}_{\mathcal{C}} \mid \psi_{\mathcal{C}}^{\xi}\right\rangle\right\} \\
= & d W .
\end{aligned}
$$

\section{APPENDIX D: THE “BEST GUESS” STATE IN THE MODIFIED JE}

The state $\tilde{\rho}_{T}$ is given by [75]

$$
\tilde{\rho}_{T}=\frac{1}{\tilde{Z}_{T}} \sum_{a} e^{-\beta\left\langle a\left|\mathrm{U}^{\dagger} \hat{H}_{\mathcal{S}}^{B} \mathrm{U}\right| a\right\rangle} \mathrm{U}|a\rangle\langle a| \mathrm{U}^{\dagger},
$$

with $\tilde{Z}_{T}=\sum_{a} \exp \left(-\beta\left\langle a\left|\mathrm{U}^{\dagger} \hat{H}_{\mathcal{S}}^{B} \mathrm{U}\right| a\right\rangle\right)$. 


\section{APPENDIX E: DISPLACED HARMONIC OSCILLATOR}

The Hamiltonian of a harmonic oscillator driven by a complex force $f(t)$ is given by

$$
\begin{aligned}
\hat{H}(t) & =\omega\left(a^{\dagger} a+\frac{1}{2}\right)+\left[f(t) a^{\dagger}+f^{*}(t) a\right] \\
& =\omega\left\{\left[a^{\dagger}+\frac{1}{\omega} f^{*}(t)\right]\left[a+\frac{1}{\omega} f(t)\right]+\frac{1}{2}\right\}-\frac{|f(t)|^{2}}{\omega} \\
& =\omega\left(A^{\dagger} A+\frac{1}{2}\right)-\frac{|f(t)|^{2}}{\omega} .
\end{aligned}
$$

$A$ and $A^{\dagger}$ fulfill the same bosonic commutation relation as $a$ and $a^{\dagger}$, and we get $\Delta F=\frac{|f(0)|^{2}-|f(T)|^{2}}{\omega}$.

As shown in Ref. [77], the dynamics under this Hamiltonian can most easily be solved for coherent states. Extending the derivation for a real force given in Ref. [77] to the case of a complex force $f(t)$ we obtain a unitary evolution

$$
\mathrm{U}(t)=e^{i \chi(t)} \hat{D}[p(t)] e^{-i \omega\left(a^{\dagger} a+\frac{1}{2}\right)},
$$

where $\hat{D}$ is the displacement operator and

$$
\begin{aligned}
& \chi(t)=-\int_{0}^{t} \operatorname{Re}\left[p(s) f^{*}(s)\right] d s, \\
& p(t)=-i e^{-i \omega t} \int_{0}^{t} e^{i \omega s} f(s) d s .
\end{aligned}
$$

Assuming, without loss of generality $f(0)=0$, the initial thermal state is diagonal in the number states of the free
Hamiltonian $\hat{H}_{0}=\omega\left(a^{\dagger} a+1 / 2\right)$. The work performed on the system starting in $|n\rangle$ is given by

$$
\begin{aligned}
\left\langle W_{n}\right\rangle= & \left\langle n\left|e^{i \hat{H}_{0}} \hat{D}^{\dagger}[p(T)]\right| \hat{H}(T)\left|\hat{D}[p(T)] e^{-i \hat{H}_{0}}\right| n\right\rangle \\
& -\langle n|\hat{H}(0)| n\rangle \\
= & \left\langle n\left|\hat{D}^{\dagger}[p(T)]\right| \hat{H}(T)|\hat{D}[p(T)]| n\right\rangle-\omega(n+1 / 2) \\
= & \left\langle n\left|\left[a^{\dagger}+p^{*}(T)\right][a+p(T)]\right| n\right\rangle+\omega / 2 \\
& +\left\langle n\left|f(T)\left[a^{\dagger}+p^{*}(T)\right]+f^{*}(T)[a+p(T)]\right| n\right\rangle \\
& -\omega(n+1 / 2) \\
= & \omega|p(T)|^{2}+2 \operatorname{Re}\left[f^{*}(T) p(T)\right] .
\end{aligned}
$$

Thus, the performed work is independent of $n$ and, therefore, $\langle W\rangle=\Delta \tilde{F}$.

The driving protocols $\theta(t)$ and $\phi(t)$ for the examples in Fig. 1(b) are

$$
\begin{aligned}
& \text { 1: } \theta(t)=\frac{\pi t}{2 T}, \quad \phi(t)=0, \\
& \text { 2: } \theta(t)=\frac{\pi t}{2 T}, \quad \phi(t)=\frac{\pi t}{2 T} \\
& \text { 3: } \theta(t)=\arcsin \frac{t}{T}, \quad \phi(t)=0, \\
& \text { 4: } \theta(t)=\arcsin \frac{t}{T}, \quad \phi(t)=\frac{2 \pi t}{T} .
\end{aligned}
$$

[11] G. Hummer and A. Szabo, Free energy surfaces from singlemolecule force spectroscopy, Acc. Chem. Res. 38, 504 (2005).

[12] N. C. Harris, Y. Song, and C.-H. Kiang, Experimental Free Energy Surface Reconstruction from Single-Molecule Force Spectroscopy Using Jarzynski's Equality, Phys. Rev. Lett. 99, 068101 (2007).

[13] J. Preiner, H. Janovjak, C. Rankl, H. Knaus, D. A. Cisneros, A. Kedrov, F. Kienberger, D. J. Muller, and P. Hinterdorfer, Free energy of membrane protein unfolding derived from single-molecule force measurements, Biophys. J. 93, 930 (2007).

[14] A. Mossa, S. de Lorenzo, J. M. Huguet, and F. Ritort, Measurement of work in single-molecule pulling experiments, J. Chem. Phys. 130, 234116 (2009).

[15] O.-P. Saira, Y. Yoon, T. Tanttu, M. Möttönen, D. V. Averin, and J. P. Pekola, Test of the Jarzynski and Crooks Fluctuation Relations in an Electronic System, Phys. Rev. Lett. 109, 180601 (2012).

[16] J. V. Koski, V. F. Maisi, T. Sagawa, and J. P. Pekola, Experimental Observation of the Role of Mutual Information in the Nonequilibrium Dynamics of a Maxwell Demon, Phys. Rev. Lett. 113, 030601 (2014).

[17] G. N. Bochkov and I. E. Kuzovlev, General theory of thermal fluctuations in nonlinear systems, Zh. Eksp. Teor. Fiz. 72, 238 (1977).

[18] S. Yukawa, A quantum analogue of the Jarzynski equality, J. Phys. Soc. Jpn. 69, 2367 (2000). 
[19] V. Chernyak and S. Mukamel, Effect of Quantum Collapse on the Distribution of Work in Driven Single Molecules, Phys. Rev. Lett. 93, 048302 (2004).

[20] A. E. Allahverdyan and T. M. Nieuwenhuizen, Fluctuations of work from quantum subensembles: The case against quantum work-fluctuation theorems, Phys. Rev. E 71, 066102 (2005).

[21] A. Engel and R. Nolte, Jarzynski equation for a simple quantum system: Comparing two definitions of work, Europhys. Lett. 79, 10003 (2007).

[22] P. Talkner, E. Lutz, and P. Hänggi, Fluctuation theorems: Work is not an observable, Phys. Rev. E 75, 050102(R) (2007).

[23] M. Campisi, P. Hänggi, and P. Talkner, Colloquium: Quantum fluctuation relations: Foundations and applications, Rev. Mod. Phys. 83, 771 (2011).

[24] A. E. Allahverdyan, Nonequilibrium quantum fluctuations of work, Phys. Rev. E 90, 032137 (2014).

[25] K. Funo and H. T. Quan, Path Integral Approach to Quantum Thermodynamics, Phys. Rev. Lett. 121, 040602 (2018).

[26] J.-J. Dong and Y.-F. Yang, Functional field integral approach to quantum work, Phys. Rev. B 100, 035124 (2019).

[27] M. Campisi, P. Talkner, and P. Hänggi, Quantum BochkovKuzovlev work fluctuation theorems, Philos. Trans. R. Soc., A 369, 291 (2011).

[28] L. Zhu, Z. Gong, B. Wu, and H. T. Quan, Quantum-classical correspondence principle for work distributions in a chaotic system, Phys. Rev. E 93, 062108 (2016).

[29] M. Perarnau-Llobet, E. Bäumer, K. V. Hovhannisyan, M. Huber, and A. Acin, No-Go Theorem for the Characterization of Work Fluctuations in Coherent Quantum Systems, Phys. Rev. Lett. 118, 070601 (2017).

[30] A. Bartolotta and S. Deffner, Jarzynski equality for driven quantum field theories, Phys. Rev. X 8, 011033 (2018).

[31] F. Binder, L. A. Correa, C. Gogolin, J. Anders, and G. Adesso, editors, Thermodynamics in the Quantum Regime: Fundamental Aspects and New Directions, Fundamental Theories of Physics, Vol. 195 (Springer International Publishing, Cham, Switzerland, 2018).

[32] K. Ito, P. Talkner, B. P. Venkatesh, and G. Watanabe, Generalized energy measurements and quantum work compatible with fluctuation theorems, Phys. Rev. A 99, 032117 (2019).

[33] G. E. Crooks, Quantum operation time reversal, Phys. Rev. A 77, 034101 (2008).

[34] P. Talkner, M. Campisi, and P. Hänggi, Fluctuation theorems in driven open quantum systems, J. Stat. Mech. (2009) P02025.

[35] M. Campisi, P. Talkner, and P. Hänggi, Fluctuation Theorems for Continuously Monitored Quantum Fluxes, Phys. Rev. Lett. 105, 140601 (2010).

[36] M. Campisi, P. Talkner, and P. Hänggi, Influence of measurements on the statistics of work performed on a quantum system, Phys. Rev. E 83, 041114 (2011).

[37] G. Watanabe, B. P. Venkatesh, and P. Talkner, Generalized energy measurements and modified transient quantum fluctuation theorems, Phys. Rev. E 89, 052116 (2014).

[38] V. Cavina, A. Mari, and V. Giovannetti, Optimal processes for probabilistic work extraction beyond the second law, Sci. Rep. 6, 29282 (2016).

[39] J. Åberg, Fully quantum fluctuation theorems, Phys. Rev. X 8, 011019 (2018).
[40] P. Strasberg, An operational approach to quantum stochastic thermodynamics, Phys. Rev. E 100, 022127 (2019).

[41] P. Strasberg and A. Winter, Stochastic thermodynamics with arbitrary interventions, Phys. Rev. E 100, 022135 (2019).

[42] A. Tobalina, I. Lizuain, and J. G. Muga, Vanishing efficiency of a speeded-up ion-in-Paul-trap Otto engine, Europhys. Lett. 127, 20005 (2019)

[43] P. Strasberg, G. Schaller, T. Brandes, and M. Esposito, Quantum and Information Thermodynamics: A Unifying Framework Based on Repeated Interactions, Phys. Rev. X 7, 021003 (2017).

[44] N. Altamirano, P. Corona-Ugalde, R. B. Mann, and M. Zych, Unitarity, feedback, interactions-Dynamics emergent from repeated measurements, New J. Phys. 19, 013035 (2017).

[45] B. P. Venkatesh, G. Watanabe, and P. Talkner, Quantum fluctuation theorems and power measurements, New J. Phys. 17, 075018 (2015)

[46] H. Tajima, N. Shiraishi, and K. Saito, Uncertainty Relations in Implementation of Unitary Operations, Phys. Rev. Lett. 121, 110403 (2018)

[47] Á. M. Alhambra, L. Masanes, J. Oppenheim, and C. Perry, Fluctuating Work: From Quantum Thermodynamical Identities to a Second Law Equality, Phys. Rev. X 6, 041017 (2016).

[48] P. Talkner and P. Hänggi, Aspects of quantum work, Phys. Rev. E 93, 022131 (2016).

[49] P. Kammerlander and J. Anders, Coherence and measurement in quantum thermodynamics, Sci. Rep. 6, 22174 (2016).

[50] J. Deng, J. D. Jaramillo, P. Hänggi, and J. Gong, Deformed Jarzynski equality, Entropy 19, 419 (2017).

[51] A. E. Rastegin, On quantum fluctuations relations with generalized energy measurements, Int. J. Theor. Phys. 57, 1425 (2018).

[52] G. Francica, J. Goold, and F. Plastina, Role of coherence in the nonequilibrium thermodynamics of quantum systems, Phys. Rev. E 99, 042105 (2019).

[53] H. Kwon and M. S. Kim, Fluctuation Theorems for a Quantum Channel, Phys. Rev. X 9, 031029 (2019).

[54] K.-D. Wu, Y. Yuan, G.-Y. Xiang, C.-F. Li, G.-C. Guo, and M. Perarnau-Llobet, Experimentally reducing the quantum measurement back action in work distributions by a collective measurement, Sci. Adv. 5, eaav4944 (2019).

[55] A similar criticism appears in Ref. [84] concerning an information-theoretic JE [85,86].

[56] C. Jarzynski, Rare events and the convergence of exponentially averaged work values, Phys. Rev. E 73, 046105 (2006).

[57] D. A. Kofke, On the sampling requirements for exponentialwork free-energy calculations, Mol. Phys. 104, 3701 (2006).

[58] S. Vaikuntanathan and C. Jarzynski, Escorted Free Energy Simulations: Improving Convergence by Reducing Dissipation, Phys. Rev. Lett. 100, 190601 (2008).

[59] J. Deng, A. M. Tan, P. Hänggi, and J. Gong, Merits and qualms of work fluctuations in classical fluctuation theorems, Phys. Rev. E 95, 012106 (2017).

[60] R. Marsland and J. England, Limits of predictions in thermodynamic systems: A review, Rep. Prog. Phys. 81, 016601 (2018).

[61] M. Campisi, R. Blattmann, S. Kohler, D. Zueco, and P. Hänggi, Employing circuit QED to measure non-equilibrium work fluctuations, New J. Phys. 15, 105028 (2013).

[62] R. Dorner, S. R. Clark, L. Heaney, R. Fazio, J. Goold, and V. Vedral, Extracting Quantum Work Statistics and Fluctuation Theorems by sIngle-Qubit Interferometry, Phys. Rev. Lett. 110, 230601 (2013). 
[63] L. Mazzola, G. De Chiara, and M. Paternostro, Measuring the Characteristic Function of the Work Distribution, Phys. Rev. Lett. 110, 230602 (2013).

[64] T. B. Batalhão, A. M. Souza, L. Mazzola, R. Auccaise, R. S. Sarthour, I. S. Oliveira, J. Goold, G. De Chiara, M. Paternostro, and R. M. Serra, Experimental Reconstruction of Work Distribution and Study of Fluctuation Relations in a Closed Quantum System, Phys. Rev. Lett. 113, 140601 (2014).

[65] A. J. Roncaglia, F. Cerisola, and J. P. Paz, Work Measurement as a Generalized Quantum Measurement, Phys. Rev. Lett. 113, 250601 (2014).

[66] G. D. Chiara, A. J. Roncaglia, and J. P. Paz, Measuring work and heat in ultracold quantum gases, New J. Phys. 17, 035004 (2015).

[67] J. P. S. Peterson, T. B. Batalhão, M. Herrera, A. M. Souza, R. S. Sarthour, I. S. Oliveira, and R. M. Serra, Experimental Characterization of a Spin Quantum Heat Engine, Phys. Rev. Lett. 123, 240601 (2019).

[68] J. M. Horowitz, Quantum-trajectory approach to the stochastic thermodynamics of a forced harmonic oscillator, Phys. Rev. E 85, 031110 (2012).

[69] J. P. Pekola, P. Solinas, A. Shnirman, and D. V. Averin, Calorimetric measurement of work in a quantum system, New J. Phys. 15, 115006 (2013).

[70] P. Solinas and S. Gasparinetti, Full distribution of work done on a quantum system for arbitrary initial states, Phys. Rev. E 92, 042150 (2015).

[71] Z. Gong, Y. Ashida, and M. Ueda, Quantum-trajectory thermodynamics with discrete feedback control, Phys. Rev. A 94, 012107 (2016).

[72] M. Naghiloo, J. J. Alonso, A. Romito, E. Lutz, and K. W. Murch, Information Gain and Loss for a Quantum Maxwell's Demon, Phys. Rev. Lett. 121, 030604 (2018).

[73] M. Naghiloo, D. Tan, P. M. Harrington, J. J. Alonso, E. Lutz, A. Romito, and K. W. Murch, Heat and Work Along Individual Trajectories of a Quantum Bit, Phys. Rev. Lett. 124, 110604 (2020).
[74] K. Micadei, G. T. Landi, and E. Lutz, Quantum Fluctuation Theorems Beyond Two-Point Measurements, Phys. Rev. Lett. 124, 090602 (2020).

[75] S. Deffner, J. P. Paz, and W. H. Zurek, Quantum work and the thermodynamic cost of quantum measurements, Phys. Rev. E 94, 010103(R) (2016).

[76] A. Sone, Y.-X. Liu, and P. Cappellaro, Quantum Jarzynski Equality of Open Quantum Systems in One-Time Measurement Scheme, Phys. Rev. Lett. 125, 060602 (2020).

[77] L. O. Castaños and A. Zuñiga-Segundo, The forced harmonic oscillator: Coherent states and the RWA, Am. J. Phys. 87, 815 (2019).

[78] T. Heinosaari and M. Ziman, The Mathematical Language of Quantum Theory: From Uncertainty to Entanglement (Cambridge University Press, Cambridge, 2011).

[79] H. Tasaki, Jarzynski relations for quantum systems and some applications, arXiv:cond-mat/0009244.

[80] J. Kurchan, A quantum fluctuation theorem, arXiv:condmat/0007360.

[81] A. E. Rastegin, Non-equilibrium equalities with unital quantum channels, J. Stat. Mech. (2013) P06016.

[82] A. E. Rastegin and K. Życzkowski, Jarzynski equality for quantum stochastic maps, Phys. Rev. E 89, 012127 (2014).

[83] S. Deffner and S. Campbell, Quantum Thermodynamics (Morgan \& Claypool Publishers, San Rafael, CA, 2019).

[84] M. Campisi and P. Hänggi, Comment on Experimental Verification of a Jarzynski-Related Information-theoretic Equality by a Single Trapped Ion, Phys. Rev. Lett. 121, 088901 (2018).

[85] V. Vedral, An information-theoretic equality implying the Jarzynski relation, J. Phys. A: Math. Theor. 45, 272001 (2012).

[86] T. P. Xiong, L. L. Yan, F. Zhou, K. Rehan, D. F. Liang, L. Chen, W. L. Yang, Z. H. Ma, M. Feng, and V. Vedral, Experimental Verification of a Jarzynski-Related Information-Theoretic Equality by a Single Trapped Ion, Phys. Rev. Lett. 120, 010601 (2018). 